\title{
Gallium nitride films grown on AIN/sapphire templates
}

Jin wu

Changchun University of Science and Technology,Wexin, Changchun, China.

High-quality GaN films were grown on AlN/c-sapphire templates by MOVPE with Hall mobility of $802 \mathrm{~cm}^{2} /$ V.s and carrier concentration of $8.1 \times 10^{16} / \mathrm{cm}^{3}$ at $300 \mathrm{~K}$ have achieved. The film low dislocation density of $5 \times 10^{7} \mathrm{~cm}^{-2}$ and X-ray rocking curve FWHM of 101 and 250 arcsec for the (0004) and (20-24) reflections were also obtained, respectively.

\section{Introduction}

Gallium nitride is an attractive material for the applications in optoelectronics, high power and high frequency electronic devices such as HEMTs, LEDs, UVLEDs, Photodectors, Solar Cells, etc.[1-5] Among many substrates, sapphire substrate dominated as one of the most popular one which has been widely used for the growth of GaN films on it. However, it is difficult to grow high quality $\mathrm{GaN}$ films because of lattice constant and thermal expansion coefficient mismatches between them. One of the most popular methods used to grown GaN/sapphire is to use thin aluminum nitride (AIN) or low temperature (LT) GaN films as the buffer layer [6,7]. The GaN films grown on sapphire substrates with LT buffer layers normally show high dislocation densities and residual stresses. In this report, we show that high quality AlN films grown on sapphire substrate at high temperature higher than $1200^{\circ} \mathrm{C}$ are excellent templates for further growth of $\mathrm{GaN}$ films. The AlN/sapphire templates are used as substrates for the further growth of $\mathrm{GaN}$ are expected to get higher crystal qualities than those grown on LT GaN layers because the AlN/sapphire templates are single crystalline. Yoshida et al. has grown GaN/AlN films at high temperatures by molecular beam epitaxy and showed that Hall mobilities and cathodoluminescence peak intensity was improved [8] even the crystal quality was not good enough. In this report, we have presented the electrical, crystal, optical qualities of GaN films grown on AlN/sapphire templates with much improvements.

\section{Experimental procedure}

Epitaxial AlN films grown on 2-in. c-plane sapphire substrates by horizontal low-pressure MOVPE. Trimethylaluminum (TMA) and ammonia $\left(\mathrm{NH}_{3}\right)$ were used as $\mathrm{Al}$ and $\mathrm{N}$ sources, respectively. Trimethylgallium (TMG) and ammonia were used as $\mathrm{Ga}$ and $\mathrm{N}$ sources, and monosilane ( $\mathrm{SiH} 4)$ was used as the n-type dopant. The substrates were heated at a temperature higher than $1200^{\circ} \mathrm{C}$ to grow approximately $3 \mu$ m-thick AlN films. Si-doped $3.2 \mu \mathrm{m}$-thick GaN films were grown on these AlN/sapphire templates without any LT buffer layers. Hall effect measurement was performed using the Van der Pauw technique. X-ray diffraction (XRD) measurement was performed to confirm the crystal quality. The surface morphology and transmission electron microscopy were applied but not shown the images here.

\section{Results and discussion}

Figure 1 shows the Hall mobilities of Si-doped $\mathrm{GaN}$ films as a function of the carrier concentration at room temperature. The highest Hall mobility were $802 \mathrm{~cm}^{2} / \mathrm{V}$.s with an electron carrier concentration of $8.1 \times 10^{16} / \mathrm{cm}^{3}$ at $300 \mathrm{~K}$. These results of $\mathrm{GaN}$ films grown on AlN/sapphire templates showed very higher values than those of GaN films grown on sapphire substrates with LT GaN as mentioned above. Thus, the GaN films grown on AlN/sapphire templates provided high quality to those grown on sapphire substrates with LT buffer layers such as LT GaN. 


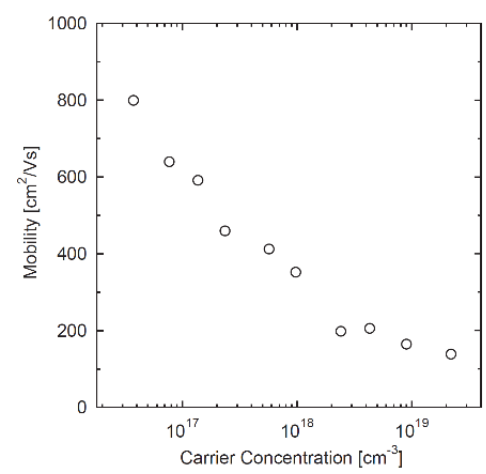

Figure 1 shows the mobility as a function of the carrier concentration.

Hall mobility and carrier concentrations of GaN films grown on AlN/sapphire templates are shown as a function of temperature as shown in Figures 2(a,b), respectively. The maximum Hall mobility was $1880 \mathrm{~cm}^{2} /$ V.s and the electron carrier concentration of $4.1 \times 10^{16} / \mathrm{cm}^{3}$ at $127 \mathrm{~K}$. As shown in the figure 2(a), the Hall mobility first increased and then decreased as the temperature decreased from 270 to $77 \mathrm{~K}$. From this temperature dependence, the main scattering mechanism was found to be acoustic phonon scattering above $127 \mathrm{~K}$. Besides that, the thermal activation energy of electrons was estimated between 77 and $270 \mathrm{~K}$ from the Figure 2(b).
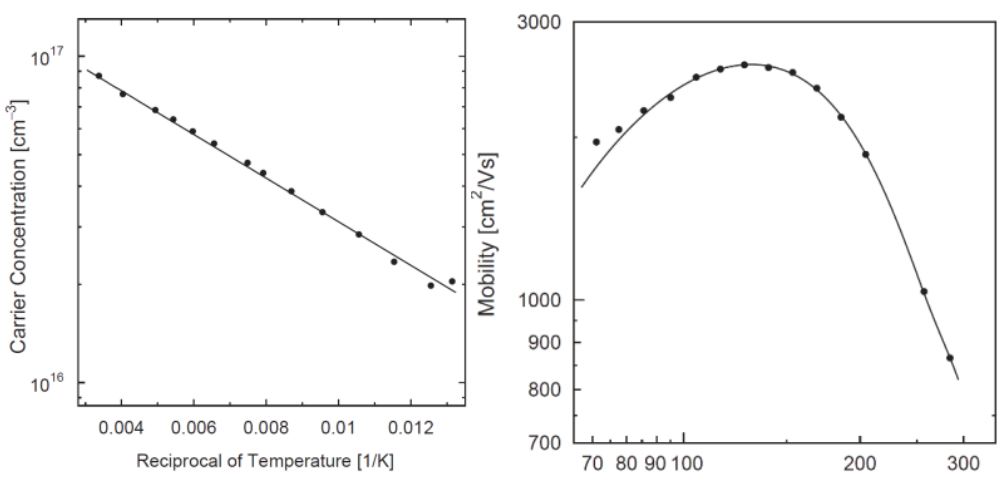

Figure 2 shows the carrier concentration and mobility of GaN.

We also carried out the AFM and TEM measurements to check the surface roughness and dislocation density. The results showed a smooth surface of the GaN film grown on an AlN/sapphire template of $0.25 \mathrm{~nm}$ and very low dislocation density $5 \times 10^{7} \mathrm{~cm}^{-2}$ together with XRD FWHM of 101 and 250 arcsec for the (0004) and (20-24) reflections (figures not shown here)

\section{Conclusions}

We have successful grown high quality GaN films on AlN/sapphire templates. Hall mobility of the GaN films were 802 and $1880 \mathrm{~cm}^{2} / \mathrm{V}$.s with electron carrier concentrations of $4.1 \times 10^{16} / \mathrm{cm}^{3}$ The XRC FWHMs were 101 and 250 arcsec for the (0004) and (20-24) reflections, respectively. AFM and TEM results showed that the crystal quality was excellent with very low surface roughness and dislocation density.

References:

1. T.-T. Luong et al., 2H-silicon carbide epitaxial growth on $c$-plane sapphire substrate using an AlN buffer layer and effects of surface pre-treatments, Electronic Materials Letters, volume 11, 352-359 (2015). 
2. T.-T. Luong et al., Performance improvements of AlGaN/GaN HEMTs by strain modification and unintentional carbon incorporation, Electronic Materials Letters, volume 11, 217-224 (2015)

3. S. Yoshida, Photoluminescence measurement of InGaN and $\mathrm{GaN}$ grown by a gas-source molecular-beam epitaxy method, Journal of Applied Physics, volume 81, 7966 (1997).

4. B. T. Tran et al., Direct Growth and Controlled Coalescence of Thick AlN Template on Micro-circle Patterned Si Substrate, Scientific Reports, volume 5, 14734 (2015)

5. B. T. Tran et al., Fabrication and characterization of n- $\mathrm{In}_{0.4} \mathrm{Ga}_{0.6} \mathrm{~N} / \mathrm{p}-\mathrm{Si}$ solar cell, Solar Energy Materials and Solar Cells, volume 102, 208-211 (2012).

6. F. Lumbantoruan et al., Investigation of TMAl preflow to the properties of AlN and GaN film grown on $\mathrm{Si}(111)$ by MOCVD, 2014 IEEE International Conference on Semiconductor Electronics (ICSE2014), 27-29 Aug. 2014, DOI: 10.1109/SMELEC.2014.6920785

7. K. L. Lin et al., Growth of GaN films on circle array patterned Si (111) substrates, Journal of Crystal Growth, Volume 401, 648-651 (2014).

8. S. Yoshida et al., Improvements on the electrical and luminescent properties of reactive molecular beam epitaxially grown GaN films by using AlN-coated sapphire substrates, Applied Physics Letters, volume 4, 427 (1983). 